\title{
The potential of programmed death ligand-1 expression in ovarian malignant germ cell tumors as a prognostic factor
}

\author{
Fadhlina Harahap, Hartono Tjahjadi, Kusmardi Kusmardi* \\ Universitas Indonesia Jakarta Pusat, Jakarta Indonesia
}

\begin{abstract}
Background: Ovarian malignant germ cell tumors (OMGCT) that fail to recover with conventional management have a poor prognosis. Several recurrent events after chemotherapy have been found. Programmed death ligand-1 (PD-L1) is expressed in various malignancies and tumor infiltrating lymphocytes (TILs) with a known role as a prognostic factor. Objective: To determine the role of PD-L1 expression in OMGCT in determining overall survival $(O S)$ and progression-free survival (PFS). Methods: Expression of PD-L1 was assessed from PD-L1 immunohistochemistry in paraffin block preparations from 40 patients diagnosed with OMGCT who met the inclusion criteria. The relationship between clinicopathological characteristics and OS and PFS was analyzed using the Kaplan-Meier method and multivariate analysis using the Cox regression model. Results: No significant relationship was found between PD-L1 expression in tumor cells with 2-year OS $(p=0.275)$ and PFS $(p=0.421)$ in OMGCT. A significant association was found between histopathologic types with 2-year OS $(p=0.002)$, and cancer stage with 2-year OS $(p=0.028)$ and PFS ( $p=0.014)$. Conclusion: PD-L1 expression in tumor cells was not related to OS and PFS in OMGCT patients. There is a tendency for death and recurrence in patients OMGCTs with low PD-L1 expression in tumor cells.
\end{abstract}

Keywords: ovarian malignant germ cell tumors, tumor infiltrating lymphocytes, programmed death ligand-1, overall survival, progression-free survival

Received: 16 $6^{\text {th }}$ June 2020; Accepted: 11 $1^{\text {th }}$ September 2020; Published: $27^{\text {th }}$ September 2020

\section{Introduction}

According to Global Cancer Incidence, Mortality and Prevalance (GLOBOCAN) 2018 data, ovarian malignancy is the ninth cause of death due to malignancy in women [1]. Data from the 2013 Indonesian cancer registry showed that ovarian malignancy ranked third among the most common malignancies [2]. Most ovarian malignancies derived from epithelial tissue, while nonepithelial malignancies only cover $5-10 \%$ of all cases. The largest group of nonepithelial malignancies is germ cell tumor $[3,4]$.

The survival and cure rates of OMGCTs are good

\footnotetext{
* Corresponding author: Kusmardi Kusmardi, Universitas Indonesia Jakarta Pusat, Jakarta Indonesia. E-mail: kusmardis@gmail.com
} 
due to their high sensitivity to chemotherapy $[3,5]$. The frequency of recurrence after chemotherapy in advanced OMGCTs is $14-20 \%$. Based on previous studies, the cure rate of OMGCTs patients is only $10 \%$ while that of testicular germ cell tumors can reach $50 \%$ [3]. The prognosis of patients who fail to recover with conventional therapy is unfavorable [6]. Therefore, research is needed to identify prognostic markers and new treatment strategies for this tumor.

Current studies are focusing on the biology of malignant tumors and its relationship with immune mechanisms [6]. One of the most recent therapies for malignancy is targeting the inhibitory checkpoint molecules [7]. Inhibitory checkpoint molecules do not target cancer cells, but rather the $\mathrm{T}$ cell receptors and ligands that weaken immune responses and increase immune tolerance [8]. Inhibitory checkpoint molecule that is explored in several research studies is programmed cell death-1 (PD-1), together with its ligand programmed death ligand-1 (PD-L1). [7] PD-1 is a member of immunoglobin superfamily that is expressed on the surface of T cells in thymus and activated cells such as T cells, natural killer cells, B cells, and monocytes. PD-L1 is expressed in organs such as placenta, heart, and activated cells such as T cells, B cells, dendritic cells, macrophages, and various tumor cells $[6,9]$. The interaction between PD-1 and PDL1 negatively regulates the immune response. Inhibition of PD-1/PD-L1 interaction can improve $\mathrm{T}$ cell responses and mediate antitumor activity. Expression of PD-L1 has been known to act as a prognostic factor in several malignancies and a predictive marker of tumor response to anti-PD-1/PD-L1 immunotherapy [10]. PDL1 expression is significantly associated with poor differentiation and overall survival (OS) in non-small cell lung carcinoma. In renal cell carcinoma, high PD-L1 expression is a negative prognostic factor that increases mortality by $81 \%$. In gastric carcinoma, PD-L1 expression is associated with large tumor size, invasion of the deep muscle layer, lymph node metastasis, and decreased patient survival. PD-L1 expression in melanoma is associated with increased aggressiveness and ease of invasion [11].

Research on ovarian clear cell carcinoma by Zhu et al. showed that high expression of PD-L1 in tumors was strongly associated with advanced stage, presence of tumor cells in ascites, resistance to platinum chemotherapy, and recurrence [12]. High expression of PD-L1 in tumors is also associated with worse OS and progression-free survival (PFS) compared to low PD-L1 expression [12]. Research on testicular germ cell tumors reported that the 5-year PFS and OS in patients with low expression of PD-L1 in tumors were $95.9 \%$ and $100 \%$, whereas the 5-year PFS and OS of patients with high PD-L1 expression were $72.2 \%$ and $72.2 \%$ [6]. A study conducted by Darb-Esfahani et al. on high-grade serous ovarian carcinoma also reported an association between PD-L1 expression with OS and PFS [8]. This study was conducted to evaluate the expression of PD-L1 in OMGCTs and assess its prognostic value in OMGCTs patients.

\section{Methods}

\section{Patients}

A total of 88 patients with a diagnosis of OMGCTs were obtained from the Department of Anatomical Pathology, Cipto Mangunkusumo National General Hospital (between 2010 and 2016). The inclusion criteria were all OMGCTs cases that had been diagnosed through histopathological examination. Exclusion criteria were cases with missing or inadequate-quality paraffin blocks, case reviews, and cases that were re-evaluated and were not eventually diagnosed as OMGCTs. The diagnosis of OMGCTs was confirmed by the pathologist by reviewing all slides blindly against the initial diagnosis. Forty patients met the inclusion criteria. 
All patients in this retrospective cohort study received informed consent. This research has been approved by the Health Research Ethics Committee of the Faculty of Medicine, Universitas Indonesia and was conducted in accordance with The Code of Ethics of the World Medical Association.

Data collection from medical records and interviews via telephone to confirm age, stage, histopathological type, lymph node metastasis, chemotherapy, and patients' conditions were carried out for two years. Staging used the 2014 International Federation of Gynecology and Obstetrics (FIGO) criteria. Initial stage is stage I including stage IA (one ovary), stage IB (both ovaries), and stage IC (one or both ovaries with malignant cells in ascites or peritoneal washings). Advanced stage is stage II, stage III, and stage IV. Histopathology is grouped into dysgerminomas and non-dysgerminomas (yolk sac tumors, immature teratomas, mature teratomas with malignant transformation, and mixed germ cell tumors). Lymph node metastases were grouped into N0 (no lymph node metastasis) and N1 (regional lymph node metastasis). Chemotherapy was categorized into receiving chemotherapy (platinum-based regimen) and not receiving chemotherapy. OS was defined as the period since the patient was diagnosed or underwent therapy until their death. PFS was defined as the period from the time a patient is diagnosed to recurrence. Recurrence could be in the form of distant metastasis, enforced by radiological examination or other investigations. We censored patients who were still alive or did not experience recurrence until the end of the observation.

\section{PD-L1 immunohistochemical staining}

Paraffin blocks were obtained from the Department of Anatomical Pathology, Cipto Mangunkusumo National General Hospital. The tissue sections were deparaffinized with xylol (3 x
3 minutes) and alcohol rehydration $(96 \%, 85 \%$, $70 \%$ ) to water and incubated in TRIS-EDTA at $96^{\circ} \mathrm{C}$ for 10 minutes within decloaking chamber to phosphate buffered saline (PBS) then peroxidase and protein blocking. Slides were incubated for 1 hour at room temperature with anti-PD-L1 monoclonal antibody (ab205921; Abcam) with 1:300 dilution to PBS and 30 minutes of incubation with the Novolink anti-rabbit immuno-peroxidase polymer secondary antibodies at room temperature to PBS. Diaminobenzidine substrate-chromogen for color reaction, hematoxylin mayer counterstain and bluing with lithium carbonate. Alcohol dehydration $(70 \%, 85 \%$, and $96 \%$ ) followed by clearing with xylol, mounting medium and covered with a deck glass. Negative and positive controls were included each time the staining was performed [6]. Positive control used for PD-L1 was placental tissue.

\section{Evaluation of PD-L1 expression}

Evaluation of PD-L1 staining was carried out in 500 tumor cells and 100 tumor infiltrating lymphocytes (TILs). Semiquantitative evaluation referred to study by Chovanec et al., which used the histoscore (H-score), a scoring system based on the percentage of the stained cells and the intensity of the stain [6]. Assessment of positivity was performed with blinding using the ImageJ software, followed by calculation of $\mathrm{H}$-score. PD-L1 expression was assessed as low (0-150) or high (160-300) [6]. The percentage of cells was expressed on a scale of $0-100 \%$. Intensity was given a score of $0-3$. Score of 0 indicated absence of PD-L1 expression, +1 score indicated the expression covered less than $10 \%$ of tumor cells, +2 score indicated moderate expression in the cytoplasm and/or cell membrane, covering $10-50 \%$ of tumor cells (Fig. 1 ), +3 score indicated strong expression in the cytoplasm and/or cell membrane, covering more than $51 \%$ of tumor cells [12]. 


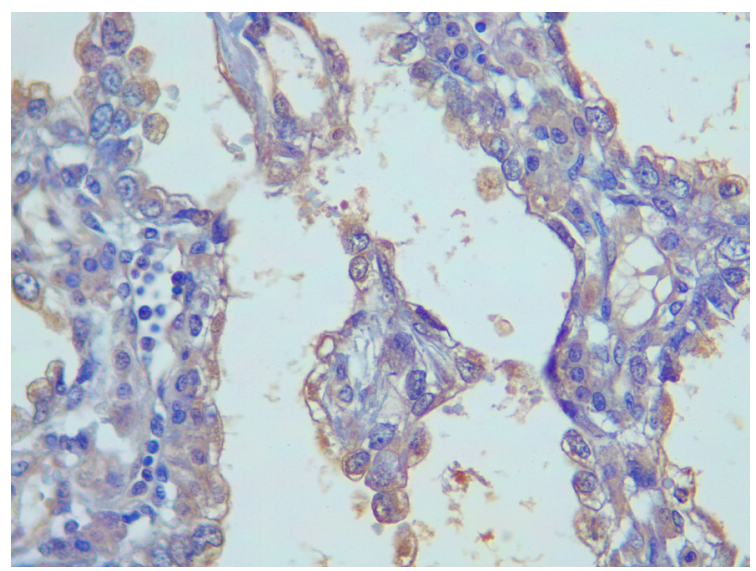

Fig. 1. PD-L1 immunohistochemical staining. Score +2 staining (moderate expression in the cytoplasm and/or cell membrane, covering 10$50 \%$ of tumor cells).

\section{Statistical analysis}

Data processing was performed using the Statistical Package for the Social Sciences software version 24. Statistical comparison between clinicopathological characteristics and PD-L1 expression, risk factors for recurrence or death, was evaluated using the Chi-square test. Fisher's test was carried out when necessary. The relationship between clinicopathological characteristics and OS and PFS was analyzed using the Kaplan-Meier method and multivariate analysis using the Cox regression model. All p-values were reported as 2 -sided, with $\mathrm{p}<0.05$ considered statistically significant.

\section{Results}

\section{Demographic and clinicopathological charac- teristics}

A total of 40 patients were included in the study (Table 1). The median age of patients was 23 years (range, 2 to 57 years). Dysgerminomas constituted 18 cases and non-dysgerminomas 22 cases. The average tumor size was $20 \mathrm{~cm}$ (range, 8 to $28 \mathrm{~cm}$ ) with the largest group being $\leq 20 \mathrm{~cm}$. Most stages were stage I (stage IA = 19 cases; stage IB $=$ not found, stage $\mathrm{IC}=8$ cases $).$ Lymph node metastasis was not found in 36 patients. Twenty nine patients did not receive chemotherapy.

Laboratory markers for ovarian malignancy or germ cell tumors such as Cancer Antigen 125 (CA-125), Lactate Dehydrogenase (LDH), or Alpha-Fetoprotein (AFP) were performed prior to surgery in only 19 out of 40 patients (Table 2). Serum CA-125 levels were found elevated in

Table 1. Patient characteristics.

\begin{tabular}{|c|c|c|}
\hline Characteristic & $n=40$ & $\%$ \\
\hline \multicolumn{3}{|l|}{ Age } \\
\hline$\leq 23$ years & 20 & 50 \\
\hline$>23$ years & 20 & 50 \\
\hline \multicolumn{3}{|l|}{ Histopathological type } \\
\hline Dysgerminoma & 18 & 45 \\
\hline Yolk sac tumors & 6 & 15 \\
\hline Immature teratomas & 9 & 22.5 \\
\hline $\begin{array}{l}\text { Mature teratomas with } \\
\text { malignant transformation }\end{array}$ & 4 & 10 \\
\hline Mixed germ cell tumors & 3 & 7.5 \\
\hline \multicolumn{3}{|l|}{ Tumor size } \\
\hline$\leq 20 \mathrm{~cm}$ & 21 & 52.5 \\
\hline$>20 \mathrm{~cm}$ & 19 & 47.5 \\
\hline \multicolumn{3}{|l|}{ Stage } \\
\hline $\mathrm{I}$ & 27 & 67.5 \\
\hline II & 2 & 5 \\
\hline III & 4 & 10 \\
\hline IV & 7 & 17.5 \\
\hline \multicolumn{3}{|l|}{ Lymph node metastasis } \\
\hline No & 36 & 90 \\
\hline N1 & 4 & 10 \\
\hline \multicolumn{3}{|l|}{ Chemotherapy } \\
\hline Yes & 11 & 27.5 \\
\hline No & 29 & 72.5 \\
\hline \multicolumn{3}{|l|}{ Recurrence } \\
\hline Yes & 4 & 10 \\
\hline No & 31 & 77.5 \\
\hline Lost to follow up & 5 & 12.5 \\
\hline \multicolumn{3}{|l|}{ Death } \\
\hline Yes & 8 & 20 \\
\hline No & 27 & 67.5 \\
\hline Lost to follow up & 5 & 12.5 \\
\hline
\end{tabular}


9 of 11 patients, 11 of 14 patients had elevated LDH levels, and 10 of 14 patients had elevated AFP levels.

\section{PD-L1 expression in tumor cells and TILs}

Thirthy six cases had a low PD-L1 expression in tumor cells, and 4 cases had a high PD-L1 expression (Table 3).

PD-L1 expression in dysgerminomas was found low in 15 cases and high in 3 cases, while in non-dysgerminomas, only one out of 22 cases showed high PD-L1 expression (Fig. 2). All 40 cases had a low PD-L1 expression in TILs (Table 3). In this study, no expression of PD-L1 was found with score of +3 .

Association between PD-L1 expression in tumor cells and TILs and clinicopathological characteristics

PD-L1 expression in tumor cells was not related to age, histopathologic type, tumor size, stage,

Table 2. Laboratory markers.

\begin{tabular}{lcccccc}
\hline \multirow{2}{*}{ Histopathological type } & \multicolumn{7}{c}{ Laboratory markers } \\
\cline { 2 - 7 } & \multicolumn{2}{c}{ CA-125 } & \multicolumn{2}{c}{ LDH } & \multicolumn{2}{c}{ AFP } \\
\cline { 2 - 7 } & Elevated & Not elevated & Elevated & Not elevated & Elevated & Not elevated \\
\hline Dysgerminomas & 4 cases & 2 cases & 6 cases & 2 cases & 6 cases & 3 cases \\
\hline Yolk sac tumors & 1 case & - & 2 cases & 1 case & 2 cases & - \\
\hline Immature teratomas & 2 cases & - & 2 cases & - & 1 case & 1 case \\
\hline $\begin{array}{l}\text { Mature teratomas with ma- } \\
\text { lignant transformation }\end{array}$ & 1 case & - & 1 case & - & 1 case & - \\
\hline Mixed germ cell tumors & 1 case & - & - & - & - & - \\
\hline
\end{tabular}

Table 3. Distribution of PD-L1 expression in tumor cells and TILs.

\begin{tabular}{lcccc}
\hline \multirow{2}{*}{ Histopathological Type } & PD-L1 expression in tumor cells & \multicolumn{2}{c}{ PD-L1 expression in TILs } \\
\cline { 2 - 5 } & Low & High & Low & High \\
\hline Dysgerminomas & 15 cases & 3 cases & 18 cases & Not found \\
\hline Yolk sac tumors & 5 cases & 1 case & 6 cases & Not found \\
\hline Immature teratomas & 9 cases & Not found & 9 cases & Not found \\
\hline $\begin{array}{l}\text { Mature teratomas with malignant } \\
\text { transformation }\end{array}$ & 4 cases & Not found & 4 cases & Not found \\
\hline Mixed germ cell tumors & 3 cases & Not found & 3 cases & Not found \\
\hline
\end{tabular}
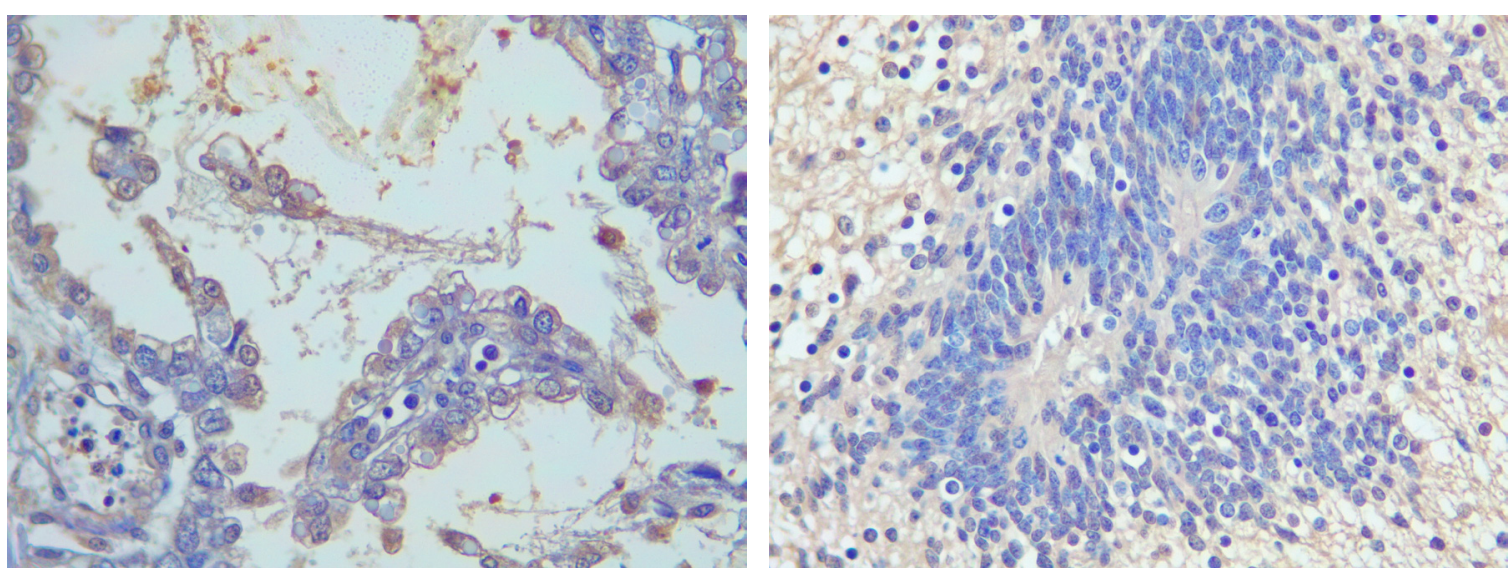

Fig. 2. PD-L1 expression in tumor cells. (A) Yolk Sac. (B) Immature Teratoma. 
lymph node metastasis, and chemotherapy (Table 4).

PD-L1 expression in TILs could not be assessed for its association with clinicopathological characteristics due to all cases having a low PD-L1 expression in TILs.

The association between PD-L1 expression in tumor cells and TILs and the laboratory markers could not be assessed either, since only for 19 out of 40 patients the laboratory tests were performed. In patients with high PD-L1 expression, serum LDH levels were found elevated in 2 patients, serum AFP levels were found elevated in 1 patient, and serum CA-125 levels were found normal. In patients with low PD-L1 expression, serum CA-125, LDH, or AFP levels could be found normal or elevated.

\section{Bivariate analysis of risk factors affecting re- currence and survival in OMGCTs}

Bivariate analysis (Table 5) indicated that PFS was related to cancer stage $(p=0.014)$. Age, his- topathologic type, tumor size, lymph node metastasis, chemotherapy, and PD-L1 expression in tumor cells were not related to PFS. It also showed association between OS and histopathological type $(p=0.002)$ and stage $(p=0.028)$. Age, tumor size, lymph node metastasis, chemotherapy, and PD-L1 expression in tumor cells were not related to OS.

Bivariate analysis could not be performed on the relationship between PFS and OS with PD-L1 expression in TILs, because all cases had low PD-L1 expression in TILs.

\section{Discussions}

One of the well-known hallmarks of cancer is the ability of tumor cells to use immunosuppressive mechanisms to avoid antitumor immune response. The interaction between PD-1/PD-L1 acts as negative regulator of T-cell-mediated antitumor immunity. PD-L1 expression is normally induced in immune cells such as macrophages

Table 4. Association between PD-L1 expression in tumor cells and clinicopathological characteristics.

\begin{tabular}{lccc}
\hline \multirow{2}{*}{ Clinicopathological characteristic } & \multicolumn{2}{c}{ PD-L1 Expression in Tumor Cells } \\
\cline { 2 - 4 } Age & Low (\%) & High (\%) & p-value \\
$\quad \leq 23$ years & $18(90)$ & $2(10)$ & 1.000 \\
$\quad>23$ years & $18(90)$ & $2(10)$ & \\
\hline Histopathological type & $15(83.3)$ & $3(16.7)$ & 0.310 \\
$\quad$ Dysgerminoma & $21(95.5)$ & $1(4.5)$ & \\
$\quad$ Non-dysgerminoma & & & \\
\hline Tumor size & $18(85.7)$ & $3(14.3)$ & \\
$\quad \leq 20$ cm & $18(94.7)$ & $1(5.3)$ & \\
$\quad>20$ cm & & & \\
\hline Stage & $23(85.2)$ & $4(14.8)$ & 0.284 \\
I & $13(100)$ & $0(0)$ & \\
II - IV & $33(91.7)$ & $3(8.3)$ & \\
\hline Lymph node metastasis & $3(75)$ & $1(25)$ & \\
N0 & & & \\
N1 & $9(81.8)$ & $2(18.2)$ & \\
\hline Chemotherapy & $27(93.1)$ & $2(6.9)$ & \\
Yes & &
\end{tabular}


Table 5. Bivariate analysis of factors associated with PFS and OS.

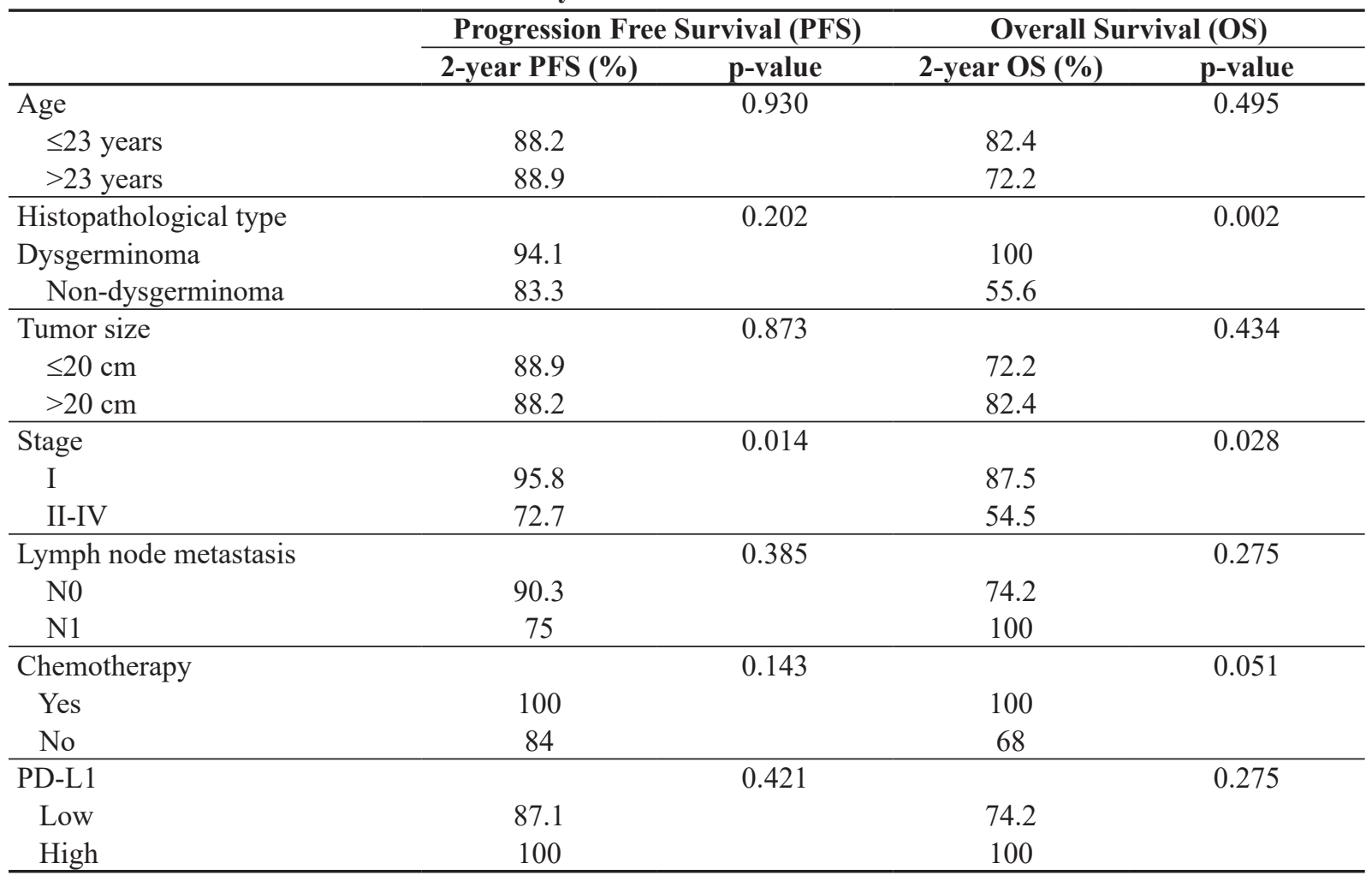

and T cells, whereas abnormal PD-L1 expression is found in several types of malignancy. Thus, a hypothesis emerges that the expression of PDL1 in tumor cells or TILs plays a role in tumor growth by inhibiting the antitumor immune response. The expression of PD-L1 on cell membrane and/or cytoplasm has been investigated in several types of malignancies. However, only a few studies have discussed germ cell tumors, especially in the ovaries. Likewise, research on PD-L1 expression and its relation to prognostic factors has not been done in ovarian germ cell tumors.

The results of this study indicated no statistically significant relationship between OS and PFS with PD-L1 expression in tumor cells in OMGCTs. This is contrary to a study by Cierna et al (clone EPR1161(2)) which reported that PFS (HR = $0.40,95 \%$ CI $0.16-1.01, \mathrm{p}=0.0081)$ and OS (HR
$=0.43,95 \%$ CI $0.15-1.23, \mathrm{p}=0.0397$ ) were better in tumors with low PD-L1 expression compared to tumors with high PD-L1 expression, and that high PD-L1 expression was found in advanced testicular germ cell tumors [9]. A study by Chovanec et al. (clone EPR1161(2)) in testicular germ cell tumors reported that the best prognosis was found in patients with low PD-L1 expression in tumor cells and high PD-L1 expression in TILs (5-year PFS and OS: $95.9 \%$ and 100\%) while the worst prognosis was found in patients with high PD-L1 expression in tumor cells and low PD-L1 expression in TILs (5-year PFS and OS: $72.2 \%$ and $72.2 \%$ ) [6].

The contradicting results found in this study could be attributed to the different location of tumors, namely in the ovaries instead of the testicles. The testicles are immunologically privileged sites, where testicular tissue is able to 
suppress immune response. Sperm maturation occurs at puberty when immune tolerance has been formed. Therefore, the immune system recognizes spermatids as a foreign matter, hence a mechanism is needed to prevent autoimmune reactions $[9,13]$. Cheng et al. reported that one of the contributing mechanisms was the interaction of PD-1/PD-L1. In their study, it was reported that PD-L1 was expressed by spermatocytes and spermatids in the seminiferous tubules. This causes the suppression of $\mathrm{T}$ cell proliferation and increases $\mathrm{T}$ cell apoptosis in the testis. Due to this interaction between PD-1/PD-L1 in normal tissue, it is important to maintain the homeostasis of the immune response to prevent autoimmunity, but in a tumor microenvironment, tumor cells can use such a mechanism to avoid immune response by deactivating cytotoxic $\mathrm{T}$ lymphocyte. It is this immune privilege of the testis mediated by PD-L1 which is thought to play a role in the pathogenesis of testicular and not ovarian germ cell tumors. [13]

In this study, 4 cases were found with high PDL1 expression in tumor cells and low PD-L1 expression in TILs. These 4 cases included 3 cases of dysgerminoma and 1 case of non-dysgerminoma (yolk sac tumor). In these 4 cases, there were no deaths or recurrences until the end of the observation. This is contrary to several previous studies which found that high PD-L1 expression in tumor cells was associated with poor OS and PFS. Dysgerminomas have better biological behavior than other types of germ cell tumors, making it plausible that dysgerminomas have better OS and PFS than other histopathological types [14]. From the results of this study, it is also known that histopathological types are statistically associated to OS. One case of yolk sac tumor in this study presented with high PDL1 expression. In this particular case, the patient had a history of chemotherapy after surgery. Absence of death or disease recurrence in this patient was likely caused by the administration of chemotherapy and might not be strongly linked to the role of PD-L1. Several studies stated that the survival rate of yolk sac tumor with chemotherapy was $80 \%$ (early stage) and 50\% (advanced stage); while several other studies stated that it ranged between $80-95 \%$ [15-17].

The absence of significant relationship between survival and PD-L1 expression in ovarian carcinoma was also reported by Drakes et al. (clone 28-8) [7]. Research on bladder malignancies performed by Davick et al. (clones SP142, 22C3, SP263) also reported that high PD-L1 expression was associated with a better OS [18]. The expression of PD-L1 also did not show any prognostic value in a study conducted by Scheel et al. (clone 5H1) in non-small cell lung carcinoma [19]. A systematic review and meta-analysis conducted by $\mathrm{Li}$ et al. on head and neck malignancy also reported no significant association between PDL1 expression and survival outcomes [20]. The same results were moreover reported by Zhong et al. in a meta-analysis of non-small cell lung carcinoma [21].

Studies on the role of PD-L1 expression as a prognostic factor have indeed been carried out. However, its role as a prognostic factor is still controversial. Several studies have reported a correlation between PD-L1 expression and survival outcomes and better clinicopathological characteristics such as histopathological type, stage, lymph node metastasis, and others. Darb-Esfahani et al. (clone EPR1161(2)) also reported an association between PD-L1 expression with OS and PFS in high-grade serous ovarian carcinoma [8]. Meta-analysis of breast malignancy carried out by Guo et al. also reported that PD-L1 expression was associated with poor prognosis, positive lymph node metastasis, poor nuclear grade, and negative estrogen receptor status [22]. Zhu et al. (clone 28-8) who conducted studies on clear ovarian cell carcinoma reported that high PD-L1 expression was associated with advanced cancer stage, presence of 
tumor cells in ascites, recurrence, resistance to platinum chemotherapy, and worse PFS and OS [12].

The discrepancies in the result of this current study and others can be caused by several things. Various kinds of PD-L1 antibody clones (E1L3N, E1J2J, SP142, 28-8, 22C3, and SP263) may cause variability in interpreting positive and negative results, because each clone has a different protein binding strength [23]. This study used the 28-8 clone that showed cytoplasmic and/or cell membrane staining. It is consistent with the research conducted by Hendry et al. which reported that significant cytoplasmic staining could be found in the $28-8$ clone and strong membrane staining could be found in the SP263 clone [24]. Discrepancies could also be caused by the absence of fixed standard in assessing PD-L1 expression, resulting in differences in interpretation. Several studies used 5\% cutoff to determine positive PD-L1 expression [25]. This study followed the research by Chovanec et al. and Zhu et al. which used $10 \%$ cutoff.

OMGCTs has very good survival rate because of its sensitivity to chemotherapy. Surgery is the main therapy in OMGCTs, followed by histopathological examination to confirm the tumor stage. Advanced OMGCTs will be recommended for chemotherapy. However, several cases still continued to recur after chemotherapy or failed to heal despite surgery and chemotherapy. This pushes the need for other therapeutic modalities that can improve survival outcomes in OMGCTs, such as checkpoint inhibitor immunotherapy (anti-PD-L1). The results of this study do not indicate an association between OS and PFS with PD-L1 expression in tumor cells and TILs. Thus, the obtained results should be interpreted with caution. However, there is a tendency for death and recurrence in OMGCTs patients with low PD-L1 expression in tumor cells. It is known that during the observation period, patients with high PD-L1 expression in tumor cells were still found alive and did not experience recurrence $(100 \%)$ while patients with low PD-L1 expression in tumor cells had death $(74.2$ $\%)$ and recurrence $(87.1 \%)$. The results of this study also showed that there were lower PD-L1 expression patterns in non-dysgerminomas patients, patients with early stages, patients who did not experience metastases, and patients who did not receive chemotherapy. But this has not reached statistical significance. Therefore, further research is needed to analyze the relationship between OS and PFS with PD-L1 expression in OMGCTs, with more balanced sample size between groups, equal numbers of cases in each histopathological type, cancer stages, lymph node metastasis, chemotherapy, and longer observation times, so that the controversy between research results can be solved.

\section{Conclusion}

No significant association was found between OS and PFS with PD-L1 expression in tumor cells in OMGCTs patients. OS was found to be associated with histopathological type and cancer stage, while PFS was found to be associated with cancer stage.

\section{Abbreviations}

GLOBOCAN - Global Cancer Incidence, Mortality and Prevalance

EUROCARE - European cancer registry-based study on survival and care of cancer patients FDA - Food and Drug Administration OMGCTS - Ovarian Malignant Germ Cell Tumor

OS - Overall Survival

PFS - Progression-Free Survival

CTLA-4 - Cytotoxic T Lymphocyte Associated-4

PD-1 - Programmed Cell Death-1

PD-L1 - Programmed Death Ligand-1

TILs - Tumor Infiltrating Lymphocytes 
FIGO - International Federation of Gynecology and Obstetrics

PBS - Phosphate Buffered Saline

H-score - Histoscore

\section{Acknowledgments}

We would like to thank the Directorate of Research and Community Engagement Universitas Indonesia for the 2019 Universitas Indonesia Research Grant.

\section{Author Contributions}

Conceptualization, F.H. and H.T.; Methodology, F.H. and K.K.; Formal Analysis, F.H. and K.K.; Investigation, F.H. and H.T.; Resources, F.H., H.T., and K.K.; Writing - Original Draft Preparation, F.H., H.T., and K.K.; Visualization, F.H. and H.T.; Supervision, H.T. and K.K.; Project Administration, F.H., H.T., and K.K.

\section{References}

1. Bray F, Ferlay J, Soerjomataram I, Siegel RL, Torre LA, Jemal A. Global cancer statistics 2018: GLOBOCAN estimates of incidence and mortality worldwide for 36 cancers in 185 countries. CA Cancer J Clin. 2018 Nov;68(6):394-424. DOI: 10.3322/caac.21492

2. Cancer Registration Agency Indonesian Association of Pathologists, Cancer in Indonesia 2013: histopathologic data. Jakarta: Cancer Registration Agency; 2017.

3. Alifrangis C, Seck MJ. Malignant ovarian germ cell tumours: an overview of management and controversies. In: Lauraine EP, Coquard IR, Lécuru F, editors. Ovarian cancers: advances through international research cooperation (gineco, engot, gcig). 1st ed. Switzerland: Springer; 2017. p.247-59. DOI: 10.1007/978-3-31932110-3_18

4. Kempf E, Desamericq G, Vieites B, Diaz-Padilla I, Calvo E, Estevez P, et al. Clinical and pathologic features of patients with non-epithelial ovarian cancer: retrospective analysis of a single institution 15-year experience. Clin Transl Oncol. 2017 Feb 1;19(2):173-9. DOI: 10.1007/s12094-016-1517-x

5. Kohli M, Zhang BY, Costello BA. Management of germ cell tumors. In: Nogales FF, Jimenez RE, editors. Pathology and biology of human germ cell tumors. Berlin: Springer; 2017. p.181-94. DOI: 10.1007/978-3662-53775-6_5
6. Chovanec M, Cierna Z, Miskovska V, Machalekova K, Svetlovska D, Kalavska K, et al. Prognostic role of programmed-death ligand 1 (PD-L1) expressing tumor infiltrating lymphocytes in testicular germ cell tumors. Oncotarget. 2017 Mar 28;8(13):21794-805. DOI: 10.18632/oncotarget.15585

7. Drakes ML, Mehrotra S, Aldulescu M, Potkul RK, Liu Y, Grisoli A, et al. Stratification of ovarian tumor pathology by expression of programmed cell death-1 (PD-1) and PD-ligand-1 (PD-L1) in ovarian cancer. J Ovarian Res. 2018 May 30;11(1):43. DOI: 10.1186/ s13048-018-0414-Z

8. Darb-Esfahani S, Kunze CA, Kulbe H, Sehouli J, Wienert S, Lindner J, et al. Prognostic impact of programmed cell death-1 (PD-1) and PD-ligand 1 (PDL1) expression in cancer cells and tumor-infiltrating lymphocytes in ovarian high grade serous carcinoma. Oncotarget. 2016 Jan 12;7(2):1486-99. DOI: 10.18632/ oncotarget.6429

9. Cierna Z, Mego M, Miskovska V, Machalekova K, Chovanec M, Svetlovska D, et al. Prognostic value of programmed-death-1 receptor (PD-1) and its ligand 1 (PD-L1) in testicular germ cell tumors. Ann Oncol. 2016 Feb;27(2):300-5. DOI: 10.1093/annonc/mdv574

10. Fankhauser CD, Curioni-Fontecedro A, Allman V, Beyer J, Tischler V, Sulser T, et al. Frequent PD-L1 expression in testicular germ cell tumors. Br J Cancer. 2015 Jul 28;113(3):411-3. DOI: 10.1038/bjc.2015.244

11. Guan J, Lim KS, Mekhail T, Chang CC, Programmed death ligand-1 (PD-L1) expression in the programmed death receptor-1 (PD-1)/PD-L1 blockade: a key player against various cancers. Arch Pathol Lab Med. 2017 Jun;141(6):851-61. DOI: 10.5858/arpa.2016-0361-RA

12. Zhu J, Wen H, Bi R, Wu Y, Wu X. Prognostic value of programmed death-ligand 1 (PD-L1) expression in ovarian clear cell carcinoma. J Gynecol Oncol. 2017 Nov;28(6):e77. DOI: 10.3802/jgo.2017.28.e77

13. Cheng X, Dai H, Wan N, Moore Y, Vankayalapati R, Dai Z. Interaction of programmed death-1 and programmed death-1 ligand-1 contributes to testicular immune privilege. Transplantation. 2009 Jun 27;87(12):1778-86. DOI: 10.1097/TP.0b013e3181a75633

14. Sever M, Jones TD, Roth LM, Karim FW, Zheng W, Michael H, et al. Expression of CD117 (c-kit) receptor in dysgerminoma of the ovary: diagnostic and therapeutic implications. Mod Pathol. 2005 Nov;18(11):1411-6. DOI: $10.1038 /$ modpathol.3800463

15. Newbould MJ. Germ cell tumors of the ovary and dysgenetic gonads. In: Wilkinson N, editor. Essentials of diagnostic gynocological pathology: pathology of the ovary, fallopian tube and peritoneum. London: Springer; 2014. p.289-326. DOI: 10.1007/978-1-4471-2942414

16. Medeiros F, Crum CP. Germ cell tumors of the ovary. In: Crum CP, Nucci MR, Lee KR, editors. Diagnostic 
gynecologic and obstetric pathology. 2nd ed. Philadelphia: Elsevier saunders; 2011. p.904-37. DOI: 10.1016/ B978-1-4377-0764-9.00029-9

17. Zaloudek CJ. Pathology of germ cell tumors. In: Soslow RA, Tornos C, editors. Diagnostic pathology of ovarian tumors. New York: Springer; 2011. p.155-91. DOI: 10.1007/978-1-4419-9751-7_13

18. Davick JJ, Frierson HF, Smolkin M, Gru AA. PD-L1 expression in tumor cells and the immunologic milieu of bladder carcinomas: a pathologic review of 165 cases. Hum Pathol. 2018 Nov;81:184-91. DOI: 10.1016/j. humpath.2018.06.028

19. Shi SJ, Wang LJ, Wang GD, Guo ZY, Wei M, Meng YL, et al. B7-H1 expression is associated with poor prognosis in colorectal carcinoma and regulates the proliferation and invasion of HCT116 colorectal cancer cells. PloS one. 2013 Oct 4;8(10):e76012. DOI: 10.1371/ journal.pone.0076012

20. Scheel AH, Ansén S, Schultheis AM, Scheffler M, Fischer RN, Michels S, et al. PD-L1 expression in nonsmall cell lung cancer: correlations with genetic alterations. Oncoimmunology. 2016 Mar 16;5(5):e1131379. DOI: $10.1080 / 2162402 X .2015 .1131379$

21. Li J, Wang P, Xu Y. Prognostic value of programmed cell death-ligand 1 expression in patients with head and neck cancers: a systematic review dan meta-analysis. PloS One. 2017 Jun 12;12(6):e0179536. DOI: 10.1371/ journal.pone.0179536

22. Guo Y, Yu P, Liu Z, Maimaiti Y, Wang S, Yin X, et al. Prognostic and clinicopathological value of programmed death ligand-1 in breast cancer: a meta-analysis. PloS one. 2016 May 26;11(5):e0156323. DOI: 10.1371/journal.pone.0156323

23. Parra ER, Villalobos P, Mino B, Rodriguez-Canales J. Comparison of different antibody clones for immunohistochemistry detection of programmed cell death ligand 1 (PD-L1) on non-small cell lung carcinoma. Appl Immunohistochem Mol Morphol. 2018 Feb;26(2):8393. DOI: 10.1097/PAI.0000000000000531

24. Hendry S, Byrne DJ, Wright GM, Young RJ, Sturrock S, Cooper WA, et al. Comparison of four PD-L1 immunohistochemical assays in lung cancer. J Thorac Oncol. 2018 Mar;13(3):367-76. DOI: 10.1016/j. jtho.2017.11.112

25. Zhu J, Wen H, Ju X, Bi R, Zuo W, Wu X. Clinical significance of programmed death ligand-1 and intra-tumoral CD8+ T lymphocytes in ovarian carcinosarcoma. PloS one. 2017 Jan 26;12(1):e0170879. DOI: 10.1371/ journal.pone. 0170879 